\title{
Editorial
}

\section{Mathematical Problems in Petri Nets Theory and Applications}

\author{
Zhiwu Li, ${ }^{1,2}$ Murat Uzam, ${ }^{3}$ GuanJun Liu, ${ }^{4}$ Georg Frey, ${ }^{5}$ and Ying Tang ${ }^{6}$ \\ ${ }^{1}$ Institute of Systems Engineering, Macau University of Science and Technology, Taipa 999078, Macau \\ ${ }^{2}$ School of Electro-Mechanical Engineering, Xidian University, Xi'an 710071, China \\ ${ }^{3}$ Elektrik-Elektronik Mühendisliği Bölümü, Mühendislik-Mimarlık Fakültesi, Melikşah Üniversitesi, Talas, 38280 Kayseri, Turkey \\ ${ }^{4}$ Department of Computer Science, Tongji University, Shanghai 200092, China \\ ${ }^{5}$ Chair of Automation and Energy Systems, Saarland University, 66123 Saarbrücken, Germany \\ ${ }^{6}$ Department of Electrical \& Computer Engineering, Rowan University, Glassboro, NJ 08028-170, USA
}

Correspondence should be addressed to Zhiwu Li; systemscontrol@gmail.com

Received 17 August 2015; Accepted 13 September 2015

Copyright (C) 2015 Zhiwu Li et al. This is an open access article distributed under the Creative Commons Attribution License, which permits unrestricted use, distribution, and reproduction in any medium, provided the original work is properly cited.

The extensive applications of communication and information technology provide spurs to the blooming of highly automated and computer-integrated systems that increasingly play an essential role in the development of the human society, leading to the deployment of discrete event systems that are thought of as a mathematical or methodological abstract of many contemporary technological systems such as intelligent urban traffic systems, automated flexible manufacturing systems, computer networks, communication protocols, logistic systems, monitoring and control of large buildings, distributed databases, and concurrent software systems. A primary challenge is to develop novel formal frameworks, analysis techniques, design tools, testing methods, and systematic control and optimization procedures for this kind of man-made highly complex systems [1], which is critical for their development and survivability. Petri nets [2] are commonly considered as such a mathematical paradigm to meet the technical requirements, as they have found extensive applications in the modeling, analysis, control [3], scheduling [4], resource management, and performance evaluation [5] of discrete event systems. This special issue focuses on the mathematical problems in Petri net theory and applications to discrete event systems.

The work by $\mathrm{C}$. Wu et al. considers the safety of rail transport that is an efficient and economic transportation mode for passengers and goods. A train rear-end collision accident is modeled with stochastic Petri nets and its quantitative analysis is then made by using a fuzzy and random approach.
A case study is reported, showing the applicability of the proposed method to the real-world train collision accidents.

Fault diagnosis and diagnosability analysis have received much attention in the discrete event system community. The work by L. Rodriguez-Urrego et al. deals with the fault diagnosis based on colored Petri nets by extending the latent nestling method for insulated-gate bipolar transistors (IGBTs) that, as a power semiconductor device, are extensively used in many modern appliances. Compared with the traditional fault diagnosis approaches using finite state machines, it avoids the state explosion problem.

The work by $\mathrm{T}$. Rak et al. reports a method of response time analysis of distributed Web systems using queueing Petri nets that are a formalism for the combined qualitative and quantitative analysis of concurrent systems. The proposed distributed Web systems modeling and design methodology have been applied in the evaluation of several system architectures under different external loads.

$\mathrm{X}$. Chen et al. consider the control problem for confusions that are an unfortunate phenomenon in discrete event systems modeled with bounded Petri nets. Two classes of confusions are proposed: conflict-increasing confusions and conflict-decreasing confusions. A control strategy is proposed for conflicts and confusions by establishing elementary conflict resolution sequences and local synchronized Petri nets, which can generate external events and event dependency relations that can be used for the control of confusions. 
By focusing on the supervisory control problem for discrete event systems in the framework of Petri nets, proposed by Y. Chen et al. is a transformation approach from nonlinear constraints to linear constraints that are usually represented by generalized mutual exclusion constraints. A nonlinear constraint can be equivalently transformed into a minimal number of conjunctive linear constraints with respect to admissible markings on the premise that the admissible marking space decided by the nonlinear constraint is convex. Then, monitors (control places) are employed to enforce the obtained linear constraints and the resulting supervisor is maximally permissive.

Different from most previous studies on deadlock control for flexible manufacturing systems in the logical level, the research by E. A. Nasr et al. investigates the analysis and evaluation of time performance of deadlock prevention policies for automated flexible manufacturing systems, including utilization of resources and system throughput, since a maximally permissive supervisor is not necessarily optimal in time performance. Taking differently sized systems as benchmark, four deadlock prevention policies are employed with different system configurations.

\section{Acknowledgments}

The editors appreciate the authors who have submitted their research work to this special issue. The editors would like to thank all reviewers for their efforts on this special issue. Unfortunately, before the publication of this issue, the lead guest editor, Professor Daniel Yuh Chao, left us forever. We are sorry that we lost such an extraordinarily distinguished Petri netter.

Zhiwu Li
Murat Uzam
GuanJun Liu
Georg Frey
Ying Tang

\section{References}

[1] S. A. Reveliotis, "Coordinating autonomy: sequential resource allocation systems for automation," IEEE Robotics \& Automation Magazine, vol. 22, no. 2, pp. 77-94, 2015.

[2] T. Murata, "Petri nets: properties, analysis and applications," Proceedings of the IEEE, vol. 77, no. 4, pp. 541-580, 1989.

[3] Z. Y. Ma, Z. W. Li, and A. Giua, "Design of optimal Petri net controllers for disjunctive generalized mutual exclusion constraints," IEEE Transactions on Automatic Control, vol. 60, no. 7, pp. 1774-1785, 2015.

[4] N. Wu, M. Zhou, and Z. Li, "Short-term scheduling of crude-oil operations: petri net-based control-theoretic approach," IEEE Robotics \& Automation Magazine, vol. 22, no. 2, pp. 64-76, 2015.

[5] J. Zhang, M. Khalgui, Z. Li, G. Frey, O. Mosbahi, and H. B. Salah, "Reconfigurable coordination of distributed discrete event control systems," IEEE Transactions on Control Systems Technology, vol. 23, no. 1, pp. 323-330, 2015. 


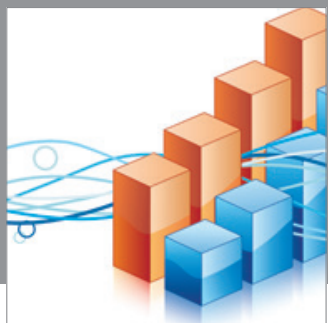

Advances in

Operations Research

mansans

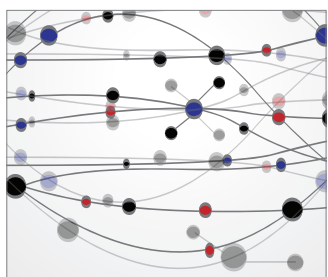

The Scientific World Journal
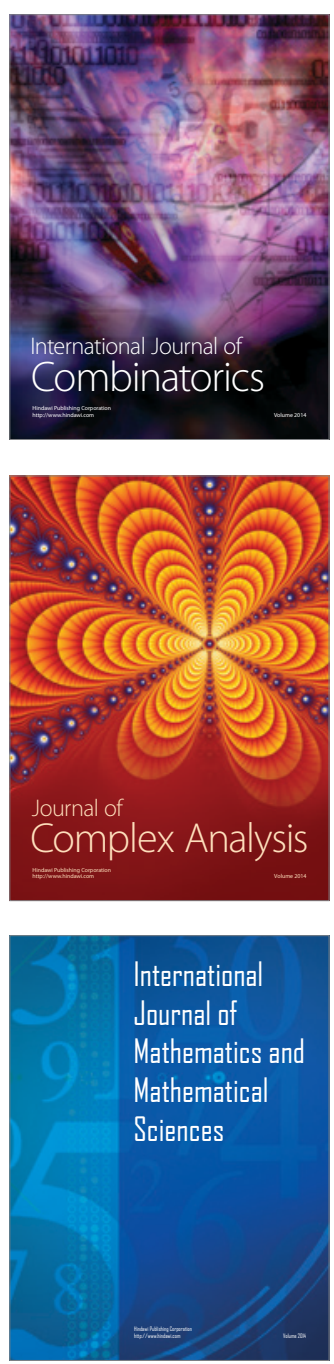
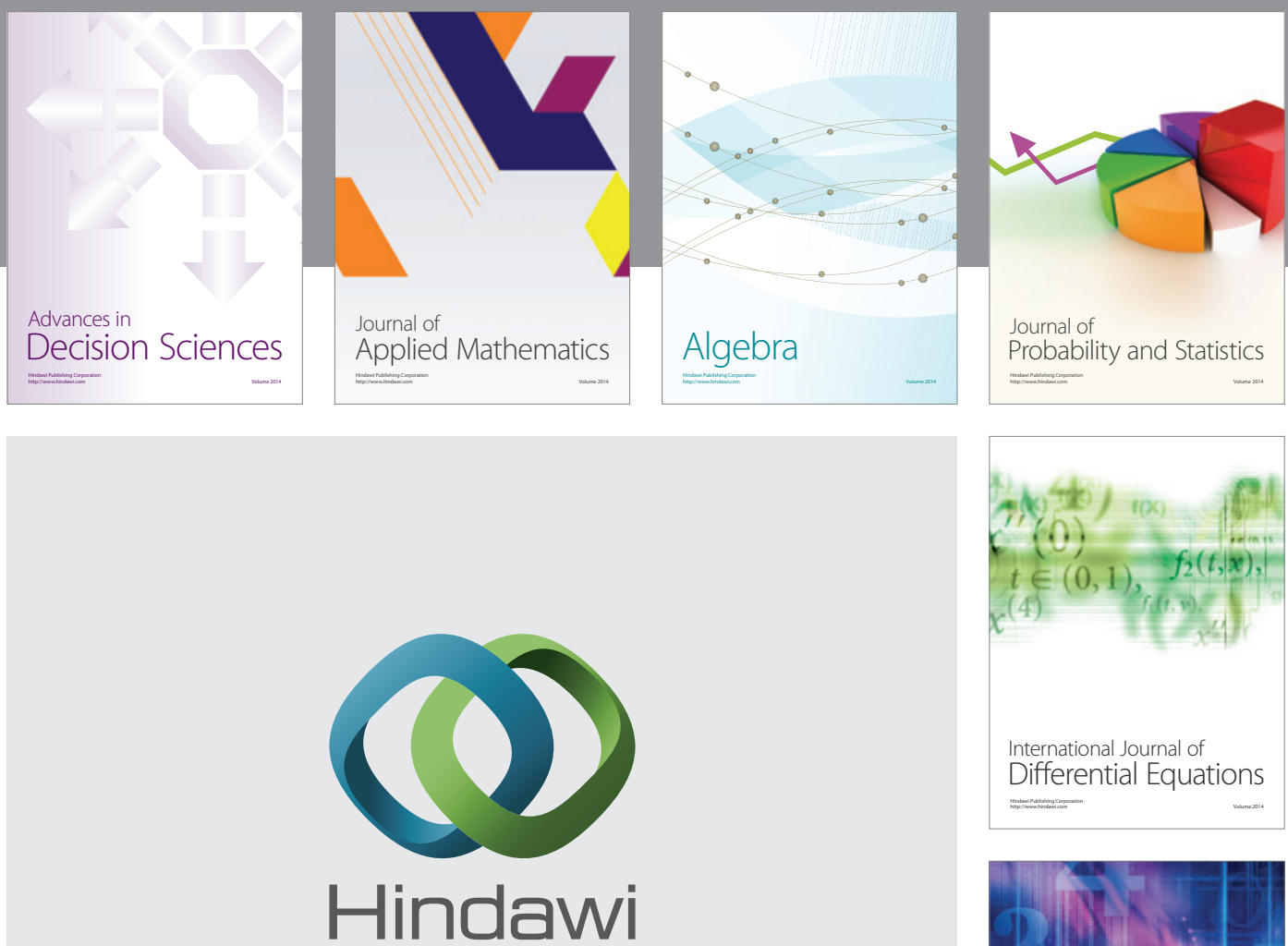

Submit your manuscripts at http://www.hindawi.com
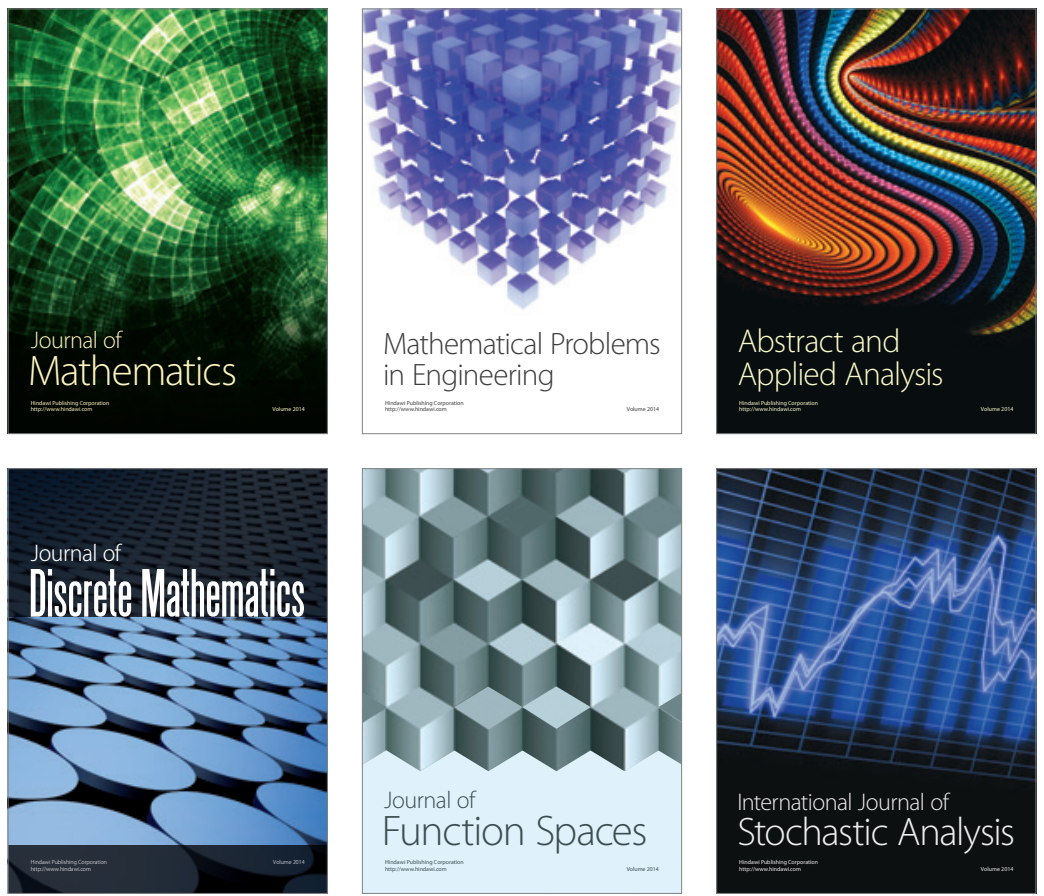

Journal of

Function Spaces

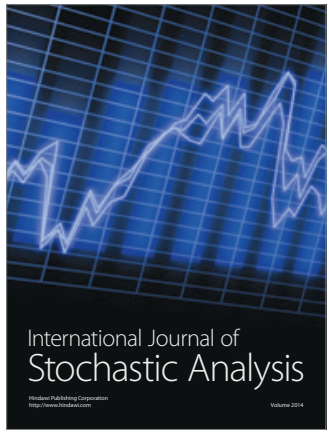

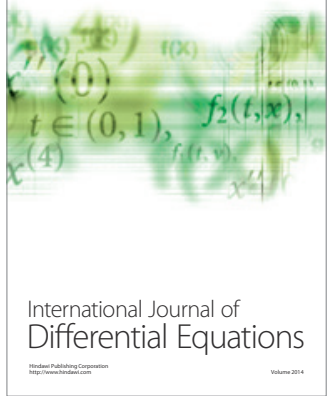
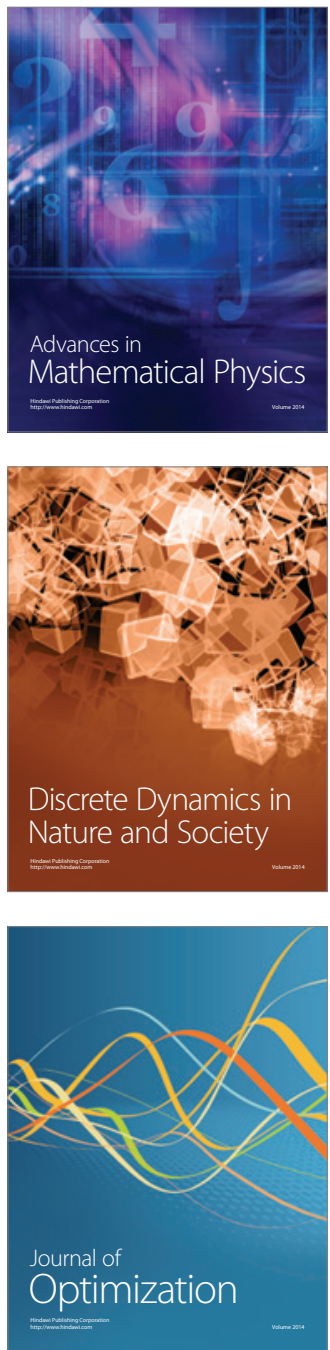